\title{
Philosophiques
}

\section{Gérard Raulet, Kant. Histoire et citoyenneté, Paris, PUF, 1996.}

\section{Maxime Lebeuf}

Volume 24, numéro 2, automne 1997

URI : https://id.erudit.org/iderudit/027463ar

DOI : https://doi.org/10.7202/027463ar

Aller au sommaire du numéro

Éditeur(s)

Société de philosophie du Québec

ISSN

0316-2923 (imprimé)

1492-1391 (numérique)

Découvrir la revue

Citer ce compte rendu

Lebeuf, M. (1997). Compte rendu de [Gérard Raulet, Kant. Histoire et citoyenneté, Paris, PUF, 1996.] Philosophiques, 24(2), 437-439.

https://doi.org/10.7202/027463ar

Ce document est protégé par la loi sur le droit d'auteur. L'utilisation des services d'Érudit (y compris la reproduction) est assujettie à sa politique d'utilisation que vous pouvez consulter en ligne.

https://apropos.erudit.org/fr/usagers/politique-dutilisation/
Cet article est diffusé et préservé par Érudit.

Érudit est un consortium interuniversitaire sans but lucratif composé de l’Université de Montréal, l'Université Laval et l'Université du Québec à Montréal. Il a pour mission la promotion et la valorisation de la recherche. https://www.erudit.org/fr/ 
Gérard Raulet, Kant. Histoire et citoyenneté, Paris, PUF, 1996.

Ce livre de Gérard Raulet amène un vent de fraîcheur sur la philosophie kantienne : à travers une relecture des opuscules sur l'histoire, l'auteur dégage une conception de l'homme dynamique, une dimension de la philosophie de Kant parfois méconnue.

L'ouvrage me semble posséder deux visées : l'une herméneutique, au sens où l'A. nous offre une interprétation des écrits kantiens sur l'histoire qu'il situe dans l'ensemble de la pensée kantienne et par rapport à ses contemporains (Herder, par exemple) ; l'autre plutôt politique : il croit que la tension actuelle entre les identités nationales et le cosmopolitisme appelle une relecture des textes fondateurs de la notion moderne de citoyenneté.

Selon moi, les principales thèses du texte se classent sous deux buts généraux. D'une part, Raulet présente les opuscules sur l 'histoire comme une application des trois Critiques fon peut presque parler, selon lui, d'une "Critique de la raison historique »), une philosophie du passage de la théorie à la pratique dont la téléologie est l'organon et le citoyen, l'acteur. Aussi, la philosophie de l'histoire y est-elle présentée comme une solution dynamique à la troisième antinomie de la raison pure (entre nécessité et liberté), dont la solution a critique "se bornait à renvoyer les adversaires dos à dos. La conception kantienne de l'histoire est également comprise comme une lutte entre Kant et les tenants de l'historiographie empirique et de la théologie ; le philosophe veut démarquer l'Histoire (où l'Idée possède une fonction heuristique) proprement dite. D'autre part, et ces arguments se classent sous la visée politique du livre, l'A. établit un parallèle entre la révolution kantienne en mé taphysique - qui passe par une distinction des territoires en fonction d' un e légalité propre (raison et entendement ne possèdent pas le même champ d 'application), où la faculté de juger apparaît comme le moyen terme permettant de passer de l'un à l'autre - et la révolution en philosophie politique basée sur la distinction de l'État de droit et la République. Kant articule ainsi nationalisme et cosmopolitisme en tenant compte des légalités propres des territoires et reconnaît un conflit durable entre l'adhésion à la République et les identités naturelles (nationales). Ici encore, c'est le jugement ou plutôt la philosophie de l'histoire qui permet de penser le passage entre les territoires.

L'ouvrage se divise en cinq chapitres, chacun offrant une relecture d'un texte kantien touchant l'histoire. Le premier chapitre s'intitule "Idée d'une histoire universelle au point de vue cosmopolitique ", le second "Conjectures sur les clébuts de l'histoire humaine ", le troisième " Les débats avec Herder et Forster sur la téléologie ", le quatrième a Sur le lieu commun : il se peut que ce soit juste en théorie, mais en pratique cela ne vaut rien " et le dernier "Le "Conflit de facultés" : Conflit de la faculté de philosophie avec la faculté de droit

Dans le chapitre premier, Raulet nous présente l'Idée d'une histoire universelle au point de vue cosmopolitique (donc l'Histoire) comme la synthèse entre connaissance physique et connaissance métaphysique. La lecture de l'A. nous montre le jugement réfléchissant découvrant $a$ posteriori dans l'histoire ce que la raison pratique y affirme a priori à travers son exigence (a priori) de l'existence du Souverain Bien. Ici, I'Idée directrice et pratique sert de principe heuristique pour dégager les sens de I 'histoire. C'est téléologiquement que le jugement trouve une régularité dans l'histoire, à travers le mécanisme de l'insociable sociabilité.

Le second chapitre aborde les Conjectures sur les débuts de l'histoire humaine. À la différence de l'Idée, ce n'est pas une application d'une Idée confrontée à l'expérience présente pour justifier le progrès. Dans les Conjectures, le commencement est confronté 
à un fil directeur relié par la raison à l'expérience. L' expérience "est notre expérience présente qui, doublée d 'une Idée régulatrice, sert de guide pour penser le début des temps. Raulet utilise ce texte pour montrer comment le mal apparait comme un aspect de la dynamique téléologique constitutive de la nature humaine. Le passage à la culture permettant l'accession à la liberté et à la raison est perçu comme la cause du mal (dont Adam est cependant responsable) : chute pour l'individu, mais progrès pour l'espèce. Sans l'opposition entre nature et raison, l'homme demeure à l'état brut, inerte.

Le chapitre trois est consacré à la méthode téléologique, organon de la philosophie de l'histoire, et à sa spécificité par rapport aux contemporains de Kant. Les textes de base sont alors le Compte-rendu des «Idées "de Herder et Sur l'emploi des principes téléologiques en philosophie. Une partie du chapitre est consacrée à la critique kantienne de la téléologie de Herder et l 'autre à un débat entre Kant et Forster sur les rapports entre observation empirique (diversité) et principe de systématisation (unité) ${ }^{1}$. Raulet centre le désaccord entre Herder et Kant sur l'articulation entre philosophie pratique (Idées) et méthode téléologique. Kant reproche à Herder une téléologie dogmatique où il fait passer pour naturel ce qui repose sur des présupposés métaphysiques. Kant n'est pas contre le dépassement du mécanisme de la nature tenté par Herder (Kant le tente lui-même), mais il critique les revendications scientifiques d'une telle entreprise, qui confond connaître et penser. Quant à Forster, le conflit porte sur la possibilité d'une Histoire qui, comme récit et non simple agrégat, est le domaine du jugement réfléchissant (téléologie) et non de la science. Les critiques adressées à Herder et Forster sont une seule et même critique, qui provient de la distinction kantienne entre phénomène et noumène et sur le statut d'une réflexion pensant leur unité.

Le chapitre quat re porte sur les rapports entretenus entre théorie et pratique. Il $s$ agit donc d'une explicitation de l'idéalisme transcendantal. Raulet parle également d'un texte de vulgarisation des Fondements de la métaphysique des mous et de la Critique de la raison pratique. On trouve également, dans ce chapitre, une explication de la polémique avec Garve (sur la possibilité du devoir), avec Hobbes (sur l'origine de la société civile) et avec Mendelssohn (sur le sens de la philanthropie et la possibilité du progrès). L'A. affirme que Kant explicite son idéalisme transcendantal : on ne peut, clans une telle perspective, disjoindre théorie et pratique. La théorie fonde la pratique, mais la connaissance dérive de l'expérience et la théorie doit s'y appliquer. Par exemple, la catégorie de la causalité * $[\ldots]$ n'exprime rien d'autre que l'idée fondamentale selon laquelle "l'expérience n'est possible que par la représentation d'une liaison nécessaire des perceptions ", c'est-à-dire la possibilité même d'une connaissance. En ce sens, les principes ramènent les phénomènes à l'unité de l'aperception "(p. 139). Les polémiques avec Garve, Hobbes et Mendelssohn portent toutes, selon lui, sur l'articulation entre théorie et pratique, entre rationnel et empirique, entre histoire pragmatique et raison pratique.

Le cinquième chapitre a pour thème la revendication philosophique à la liberté cle penser dans tous les domaines, même ceux des "facultés supérieures": la théologie, le droit et la médecine. Le conflit avec la faculté de droit concerne le droit à la publicité des écrits philosophiques et revendique l'indépendance d'une a loi philosophique": la loi morale. L'A. y discute également la "preuve historique kantienne d'une disposition morale en I'homme, fondant alors l'existence d'une loi morale. Cette a preuve " est l 'enthousiasme général des Européens de l'époque vis-

1. Gérard Raulet a par ailleurs déjà fait paraître un livre complet sur la question des rapports entre Kant et ses contemporains et sur l'ensemble des Lumières allemandes. Voir Gérard Raulet, L'Aufklarung. Les Lumiëres allemandes, Paris, 1995. 
à-vis de la Révolution française qui, précisément, est réalisée au nom du droit naturel, de la « loi philosophique ". Selon Kant, il s'agit d'un * signe • d'une disposition morale en l'homme puisque l'enthousiasme est selon lui désintéressé et universel. L'A. ajoute que ce a signe phénoménal (sensible) d'une réalité nouménale (intelligible) établit un pont entre le point de vue empirique et le point de vue transcendantal. Selon l'auteur, cet enthousiasme bien particulier ne serait rien de moins que l'expérience du sublime.

Pour finir, Kant : Histoire et citoyenneté me semble un texte fort important pour quiconque désire se faire une idée juste de la philosophie de Kant. De plus, l'ouvrage offre des éclaircissements importants sur le statut de la téléologie et du citoyen ainsi que sur son rapport à la philosophie critique. Toutefois, la téléologie est l'organon non seulement de la philosophie kantienne de l'histoire, mais aussi de sa philosophie de l'éducation, qui sert de modèle à la philosophie de l'histoire. Enfin, contrairement à l'opinion courante, ses écrits sur l'histoire ne sont pas un appendice aux Critiques, mais bien leur résultat : la " philosophie scolastique " des trois Critiques doit déboucher sur une a philosophie cosmique " dont, aux dires de Kant lui-même, la "philosophie critique "n'est que le préambule, chose que Raulet a le mérite de nous faire remarquer.

\author{
Maxime Lebeuf \\ Département de philosophie \\ Université du Québec à Trois-Rivières
}

